\title{
The Ultrasmall Biocompatible CuS@BSA Nanoparticle and Its Photothermal Effects
}

\begin{abstract}
Xiaofang Wan ${ }^{1,2}$, Maixian Liu',2, Mingze Ma ${ }^{1}$, Danyang Chen ${ }^{1}$, Na Wu ${ }^{3}$, Li Li ${ }^{1,4}$, Zhongjun Lij, Guimiao Lin ${ }^{4}$, Xiaomei Wang ${ }^{4 *}$ and Gaixia Xu',2*

${ }^{1}$ National-Regional Key Technology Engineering Laboratory for Medical Ultrasound, Guangdong Key Laboratory for Biomedical Measurements and Ultrasound Imaging, Department of Biomedical Engineering, School of Medicine, Shenzhen University, Shenzhen, China, ${ }^{2}$ Key Laboratory of Optoelectronics Devices and Systems of Ministry of Education/Guangdong Province, College of Optoelectronic Engineering, Shenzhen University, Shenzhen, China, ${ }^{3}$ Carson International Cancer Center, School of Medicine, Shenzhen University, Shenzhen, China, ${ }^{4}$ Department of Physiology, School of Basic Medical Sciences, Shenzhen University, Shenzhen, China, ${ }^{5}$ Collaborative Innovation Center for Optoelectronic Science and Technology, College of Optoelectronic Engineering, Shenzhen University, Shenzhen, China
\end{abstract}

\section{OPEN ACCESS}

Edited by:

Eleonore Fröhlich,

Medical University of Graz, Austria

Reviewed by:

Madhappan Santha Moorthy,

Pukyong National University,

South Korea

Guosheng Song,

Hunan University, China

${ }^{*}$ Correspondence:

Xiaomei Wang

xmwang@szu.edu.cn

Gaixia Xu

xugaixia@szu.edu.cn

Specialty section:

This article was submitted to

Predictive Toxicology,

a section of the journal

Frontiers in Pharmacology

Received: 15 November 2018

Accepted: 06 February 2019

Published: 26 February 2019

Citation:

Wan X, Liu M, Ma M, Chen D,

Wu N, Li L, Li Z, Lin G, Wang X and

$X u G$ (2019) The Ultrasmall

BiocompatibleCuS@BSA

Nanoparticle and Its Photothermal

Effects. Front. Pharmacol. 10:141.

doi: 10.3389/fphar.2019.00141
Nanomaterials with localized surface plasmon resonance (LSPR) have exquisite optical properties, which allow a wide range of applications. Non-stoichiometric copper sulfides with active LSPR have drawn great attention, because its LSPR peak falls in the NIR region that is suitable for deep bioimaging and photothermal therapy (PTT). Despite numerous biomedical applications, the biocompatibility and toxicity of copper sulfides have not been studied systematically. In this contribution, we synthesized the ultrasmall biocompatible copper sulfide nanoparticle encapsulated within bovine serum albumin (BSA), CuS@BSA. The physical features of CuS@BSA were characterized. The MTT and flow cytometry assays were performed. The in vitro PTT was also investigated. The results indicated that such CuS@BSA nanoparticle had an average TEM size of $8 \mathrm{~nm}$, and an average DLS size of $15 \mathrm{~nm}$. A lower concentration of CuS@BSA was not toxic to HeLa cells, but the critical apoptotic events occurred in HeLa cells after co-incubation with $45 \mu \mathrm{g} / \mathrm{mL}$ CuS@BSA for $48 \mathrm{~h}$. The photothermal effect of the CuS@BSA in aqueous medium were concentration-dependent and time-dependent, which were also verified by flow cytometry and microscopy, while the CuS@BSA were co-cultured with HeLa cells and treated with laser. This work designed an ultrasmall potential biocompatible nanoparticle, CuS@BSA, for cancer photothermal therapy, and provided the toxic information to safely guide its biomedical applications.

Keywords: CuS@BSA, phototherma, in vivo, toxicity, HeLa cells

\section{INTRODUCTION}

With the rapid development of nanotechnology, many kinds of nanomaterials with unique physical and chemical properties have been designed, synthesized and applied in various fields (Liu and Swihart, 2014). Unique phenomena observed in nanomaterials (Dutta and Dolui, 2008), such as surface plasmon resonance (SPR) (Zhu and Su, 2013) and localized surface plasmon resonance (LSPR) (Choi et al., 2011), are particularly important optical properties that are increasingly playing 
important roles in biomedical and optoelectronic applications (Ozbay, 2006; Dreaden et al., 2012). In particular, the nanoparticles with high near-infrared absorption/emission are helpful for deep in vivo theranostics, such as photothermal therapy (PTT) and bioimaging (Dreaden et al., 2012). Generally, besides the surgery, radiotherapy and chemotherapy are most commonly accepted by clinical cancer therapy (Liu et al., 2013). However, it is well know that these methods could destroyed the tumor, as well as the normal cells and the immune system, which caused more serious consequences to patients (Kievit and Zhang, 2011). Fortunately, novel tumor therapeutic strategies, such as immunological therapy, biological therapy, PTT, and photodynamics therapy, have given hope to patients, because of the high selectivity, minimal invasion, and adverse effects (Choi et al., 2011; Zhang et al., 2015).

In recent years, more and more photothermal materials have been studied by researchers, such as $\mathrm{Au}, \mathrm{Ag}$ and Pd-based novel metal nanoparticles. However, long-term safety remains a concern, which impedes them from being widely used in clinical applications (Nel et al., 2006; Liu et al., 2013). Thus, developing PTT agents that consist of naturally occurring substances in organisms would be highly beneficial for in vivo applications. Because such agents can effectively avoid serious adverse effects caused by the long-term retention of foreign substances in patients, and biodegradation through metabolism (Liu et al., 2013). Benefiting from the high optical extinction coefficient in the near-infrared region, various gold nanostructures, including gold nanoparticles (Geng et al., 2012), nanorods (Huang et al., 2010), nanostars (Yuan et al., 2012), nanocage (Yavuz et al., 2009), and nanoshells (Liu et al., 2011), have become the most popular photothermal agents in near-infrared PTT. Nevertheless, the high cost, long retention, and shape-dependent photothermal effects, limited their further applications. Some two dimensional materials, including $\mathrm{Bi}_{2} \mathrm{Se}_{3}$ (Song et al., 2016), $\mathrm{WO}_{3}$ (Balandeh et al., 2015), graphene (Chen et al., 2015), and black phosphorus (Jiang et al., 2016) have also been studied by researchers. Recently, the alternative agent of $\mathrm{Au}$, copper sulfide nanoparticles ( $\mathrm{CuS}$ NPs), have been investigated widely by researchers due to their outstanding absorbance, effective thermal conversion, and simple preparation (Goel et al., 2014; Liang et al., 2018). In particular, CuS NPs with strong NIR absorbance and photo stability, are ideal candidates for in vivo applications (Zhou et al., 2010). However, despite the positive results for in vitro and in vivo biomedical applications, we know little about the toxicity of $\mathrm{CuS}$ nanostructures. Zhang et al. (2015) found that the BSA$\mathrm{CuS}$ nanoparticle had a cell viability of $90 \%$ at a concentration of $230 \mathrm{mg} / \mathrm{L}$. Liang et al. (2018) performed a long-term toxicity study with a large dose of CuS@MSN and the results showed only minimal toxicity for living female BALB/c mice. Feng et al. (2015) studied the toxicity of CuS nanosheets for different types of cells. They found that nanosheets was non-toxic to HeLa, $\mathrm{KB}$, and RAW 264.7 cells, while the nanosheets concentrations were below $50 \mu \mathrm{g} / \mathrm{mL}$, and the cell viability decreased while the concentration was above $100 \mu \mathrm{g} / \mathrm{mL}$. However, the more systematical toxicity study should be performed to advance the PTT application of CuS. A better understanding of the toxicity characteristics of CuS nanostructures will facilitate the development of a safe and effective PTT agent for cancer therapy (Liang et al., 2018).

In this work, we synthesized an ultrasmall copper sulfide nanoparticle encapsulated in BSA. The MTT assay and flow cytometry assay were performed to analyze the toxicity of such CuS@BSA nanoparticle. PTT was performed and considerable cell apoptosis was observed after irradiating the cells with a $1064 \mathrm{~nm}$ laser for $6 \mathrm{~min}$. The flow cytometry and microscopy were used to investigate the PTT efficiency quantitatively and qualitatively, respectively. The results showed that the nanoparticle inhibited cell proliferation at a concentration of $45 \mu \mathrm{g} / \mathrm{mL}$ for $48 \mathrm{~h}$ co-incubation. The PTT was concentration-dependent and time-dependent. These results suggest that optimization of concentration and irradiation time are necessary for the safety of CuS@BSA nanoparticle in biomedical applications.

\section{MATERIALS AND METHODS}

\section{Chemicals}

All chemicals and reagents used were analytical grade. Ultrapure water (Hangzhou Wahaha Group Co., Ltd., (Hangzhou, China) was used throughout this work. BSA was obtained from Yan cheng Sai bao Biotechnology Co., Ltd., (Jiangsu, China). $\mathrm{CuCl}_{2} \cdot 2 \mathrm{H}_{2} \mathrm{O}$ and $\left(\mathrm{NH}_{4}\right)_{2} \mathrm{~S}$ were purchased from MACKLIN Co., Ltd., (Shanghai, China).

\section{Synthesis}

The CuS@BSA nanoparticle was prepared by a simple method. Firstly, $100 \mathrm{mg}$ of BSA was dissolved in $10 \mathrm{~mL}$ ultrapure water, and then the $250 \mu \mathrm{L} \mathrm{CuCl} 2 \cdot 2 \mathrm{H}_{2} \mathrm{O}$ solution at a concentration of $0.75 \mathrm{M}$ was added to the above solution under magnetic stirring. Once the color of the mixture changed into blue, $100 \mu \mathrm{L}$ of the $\left(\mathrm{NH}_{4}\right)_{2} \mathrm{~S}$ solution at a concentration of $3.2 \mathrm{M}$ was added quickly. The mixture solution was subsequently heated up to $90^{\circ} \mathrm{C}$ for $30 \mathrm{~min}$ until the color turned to dark green, which indicated the formation of the CuS@BSA nanoparticle. The obtained CuS@BSA nanoparticle were stored at $4^{\circ} \mathrm{C}$. The CuS@BSA nanoparticle used in the following experiments were dissolved in a PBS solution and filtered through a $220 \mathrm{~nm}$ film.

\section{Characterization}

The morphology and microstructure of the CuS@BSA nanoparticle were characterized with a transmission electron microscope (TEM) (TecnaiG2 F20 S-TWIN, FEI, United States) operating at an accelerating voltage of $200 \mathrm{kV}$ at room temperature. The samples for TEM were prepared by depositing a diluted nanoparticle solution into a 230-mesh $\mathrm{Cu}$ grid, sample droplets dried by water dispersion. The absorption spectra of CuS@BSA were measured by UV-vis (Lambda 750, PE, United States). The hydrodynamic diameters and size distribution of nanoparticles were characterized by dynamic light scattering technology on a Malvern Zetasizer (Zetasizer Nano ZS, Malvern, United Kingdom). CuS@BSA nanoparticle was diluted by ultrapure water before measurement. 


\section{Colloidal Stability and Photothermal Performance}

The stability of the BSA@CuS nanoparticle in the various media, such as pure water, PBS $(\mathrm{pH}=7.4)$, FBS, and DMEM, was tested. The photothermal-induced temperature elevation was quantified by inserting a thermos-couple into the solution of nanoparticle irradiated with a $1064 \mathrm{~nm}$ laser. The solutions of the CuS@BSA nanoparticle with different concentrations $(22.5,45,90$, and $180 \mu \mathrm{g} / \mathrm{mL}$ ) were irradiated with the laser at a power density of $0.73 \mathrm{~W} \mathrm{~cm}^{-2}$ for $3 \mathrm{~min}$ to evaluate the photothermal performance.

\section{Cell Cultures}

The human cervical carcinoma cells, HeLa, were obtained from American Type Culture Collection (ATCC) and cultured in Dulbecco's Modified Eagle's Medium (DMEM, Gibco, United States), supplemented with $10 \%$ fetal bovine serum (FBS, Gibco, United States) and 100U penicillin/streptomycin (Gibco, United States). All cells were cultured at $37^{\circ} \mathrm{C}$ in a humidified atmosphere with $5 \% \mathrm{CO}_{2}$.

\section{MTT Assay for BSA@CuS Nanoparticle}

The cell viability of HeLa cells was evaluated through an MTT (Sigma-Aldrich, United States) assay. The cells were seeded into a 96-well plate $\left(5 \times 10^{3}\right.$ cells/well $)$ and incubated for $24 \mathrm{~h}$ at $37^{\circ} \mathrm{C}$ under $5 \% \mathrm{CO}_{2}$. The cells were subsequently co-incubated with different concentrations of the CuS@BSA nanoparticle for $24 \mathrm{~h}$ or $48 \mathrm{~h}$. The MTT solution $(5 \mathrm{mg} / \mathrm{mL})$ was added into cells for $10 \mu \mathrm{L} /$ well. After $4 \mathrm{~h}$ of incubation, the culture medium was removed carefully and DMSO $(150 \mu \mathrm{L} /$ well $)$ was added to sufficiently dissolve the blue crystals. The plates were assayed by a micro plate reader (Multiskan FC, Thermo Fisher, Finland) at a wavelength of $570 \mathrm{~nm}$. The cell viability was calculated by normalizing the absorbance of the treated wells vs the control wells.

\section{Cell Apoptosis Detected by Flow Cytometry}

The cell apoptosis was measured with the Annexin V-FITC Apoptosis Detection Kit (BD PharmingenR United States). On the day before assay, cells were planted onto 6-well plates $\left(10 \times 10^{4}\right.$ cells/well $)$ and incubated for $24 \mathrm{~h}$ at $37^{\circ} \mathrm{C}$ under $5 \%$
A

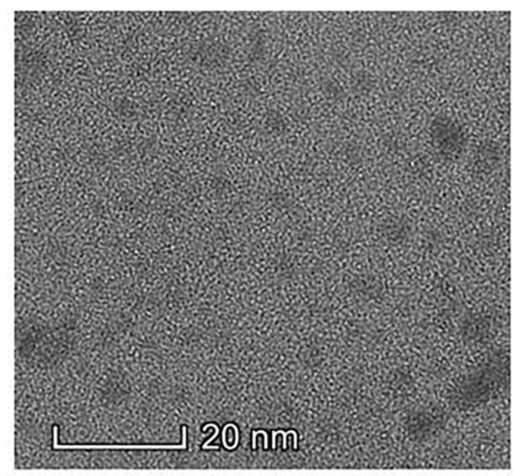

B

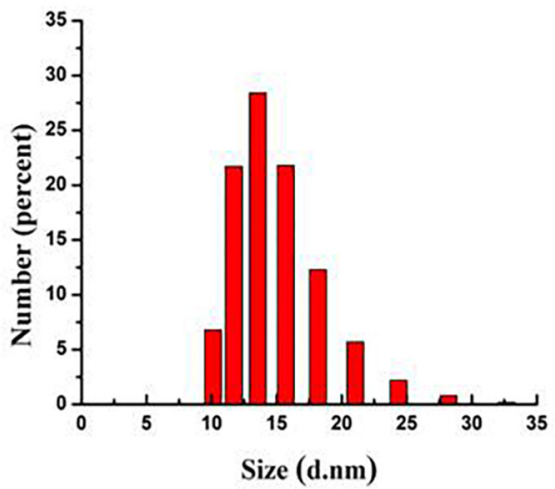

C

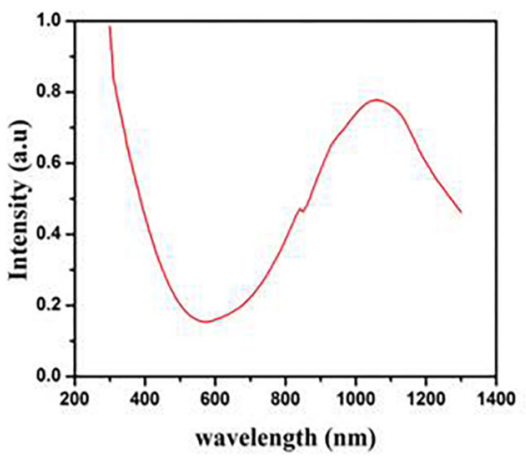

FIGURE 1 | The Characterization of the CuS@BSA nanoparticle. (A) A TEM image. Scale bar: 20 nm. (B) DLS analysis. (C) Absorbance spectrum.

A

$24 \mathrm{H}$

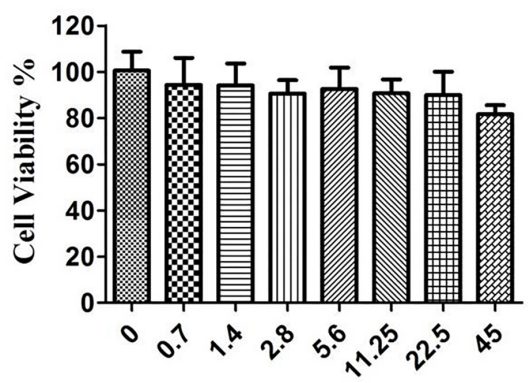

Concentration of $(\mu \mathrm{g} / \mathrm{mL})$

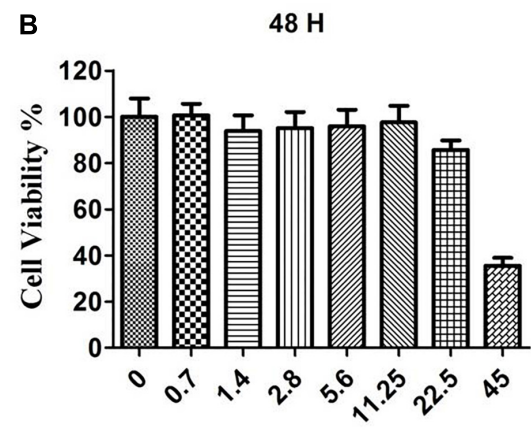

Concentration of $(\mu \mathrm{g} / \mathrm{mL})$

FIGURE 2 | The cytotoxicity of CuS@BSA nanoparticle for HeLa cells. (A) The HeLa cells were co-cultured with the CuS@BSA nanoparticle for 24 h. (B) The HeLa cells were co-cultured with the CuS@BSA nanoparticle for 48 h. $n=6$. 
$\mathrm{CO}_{2}$. For apoptosis detection, the experimental groups were cocultured with CuS@BSA nanoparticle for 24 or 48 h, while the blank groups were treated with PBS. All cells were dissociated with a trypsin solution (Gibco, United States) without EDTA and collected by centrifugation at $1000 \mathrm{rpm}$ for $5 \mathrm{~min}$. After washed with pre-cooled PBS, cells were resuspended in $100 \mu \mathrm{L}$ $1 \times$ binding buffer. A 5 microliter Annexin V-FITC solution was added into cells and cells were incubated for $5 \mathrm{~min}$ at room temperature and protected from light. Finally, $10 \mu \mathrm{L}$ propidium iodide (PI) solution and $400 \mu \mathrm{L}$ PBS was added into cells. The signals of FITC fluorescence were detected by a flow cytometer (FACSAria II, BD, United States).

\section{In vitro Photothermal Therapy of CuS@BSA Nanoparticle}

On the day before assay, cells were planted onto 6 -well plates $\left(10^{4}\right.$ cells/well) and incubated for $24 \mathrm{~h}$ at $37^{\circ} \mathrm{C}$ under $5 \% \mathrm{CO}_{2}$. HeLa cells were treated without or with the CuS@BSA nanoparticle, and exposed to the $1064 \mathrm{~nm}$ laser with a power density of $0.55 \mathrm{~W}$ $\mathrm{cm}^{-2}$ for 0-5 min. The dyes, Calcein-AM and PI, were used to stain the population of viable and necrotic cells, respectively. The Calcein-AM was incubated with cells for $30 \mathrm{~min}$ at $37^{\circ} \mathrm{C}$. Later, the cells were washed three times with PBS to remove the CalceinAM dye. Finally, $10 \mu \mathrm{L}$ PI was added into cells samples at a final volume of $100 \mu \mathrm{L}$.

\section{Data Statistics}

MTT experimental data were expressed as mean \pm standard deviation (SD). Multigroup comparisons of the means were carried out through a one-way analysis of a variance (ANOVA) test.

\section{RESULTS AND DISCUSSION}

\section{Characterization of BSA-CuS Nanoparticle}

The morphology of the CuS@BSA nanoparticle were shown as a TEM image (Figure 1A). It demonstrated a relatively monodispersed size distribution with an averaged size of $8 \mathrm{~nm}$. The hydrodynamic diameters of the aqueous nanoparticle were $15 \mathrm{~nm}$ (Figure 1B). The NIR absorption of the CuS@BSA nanoparticle were measured by UV-vis, and the absorption peak was at around $1060 \mathrm{~nm}$ (Figure 1C). The molar extinction coefficient is $7.06 \times 10^{9} \mathrm{~cm}^{-1} \mathrm{M}^{-1}$.

\section{Cytotoxicity of CuS@BSA Nanoparticle}

In order to evaluate the cytotoxicity of the CuS@BSA nanoparticle, HeLa cells were co-incubated with a CuS@BSA nanoparticle at different concentrations for $24 \mathrm{~h}$ and $48 \mathrm{~h}$. The standard MTT assay was carried out to determine the

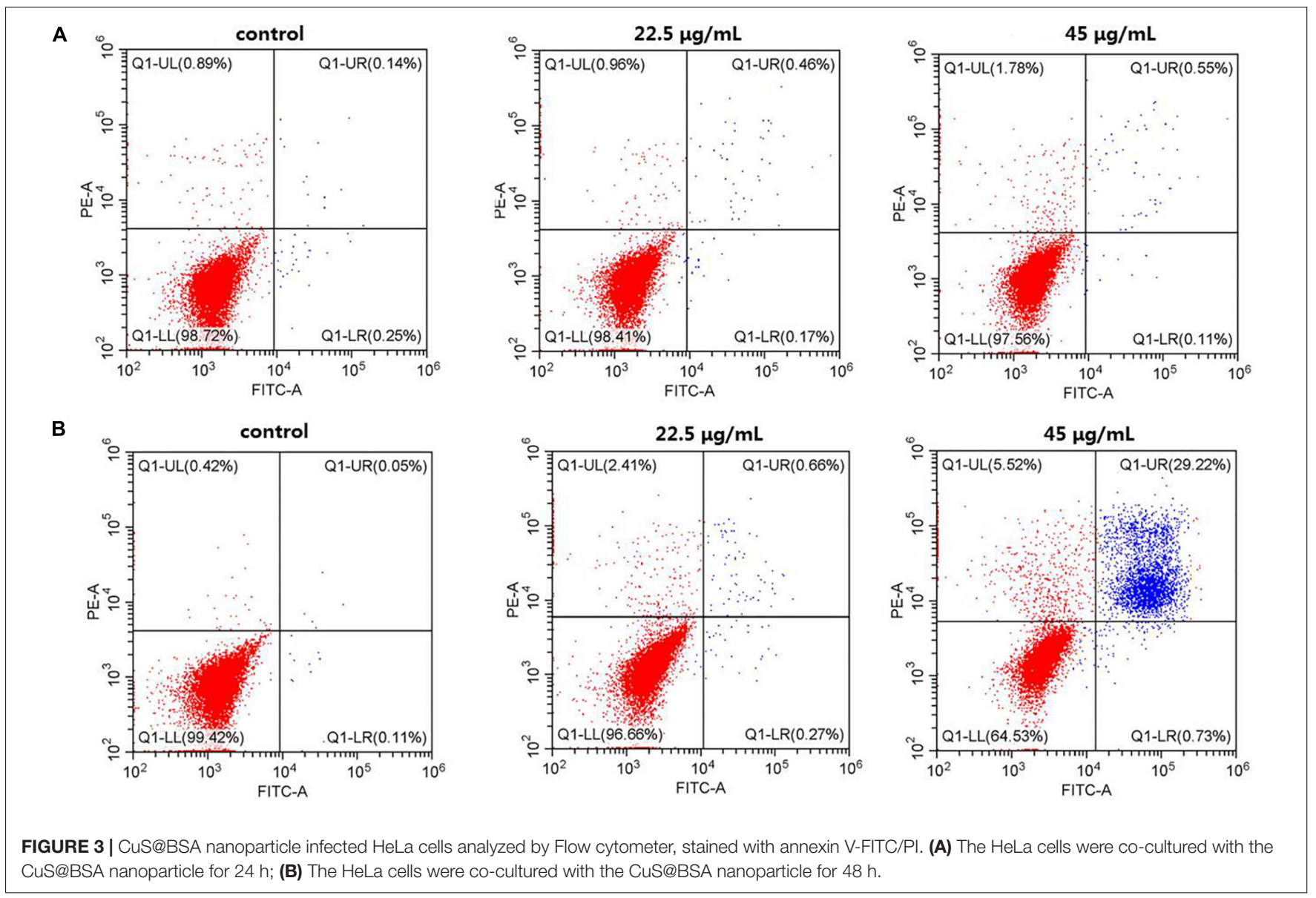



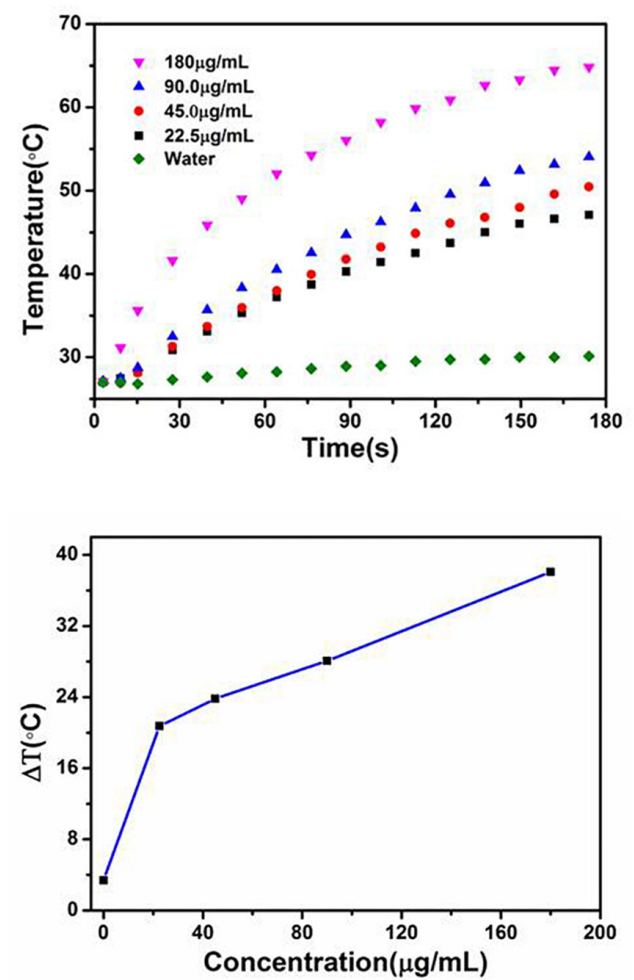

B

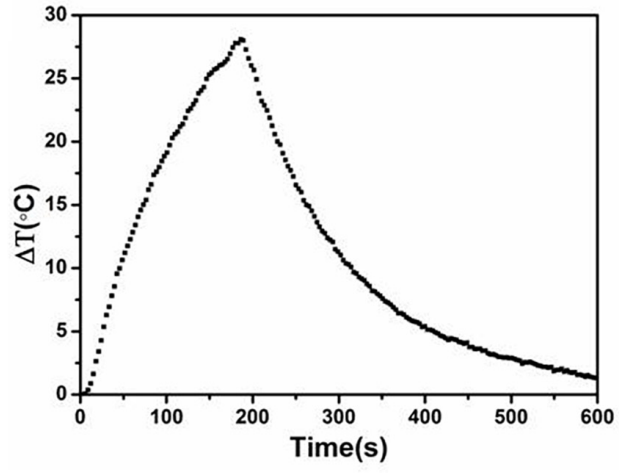

D
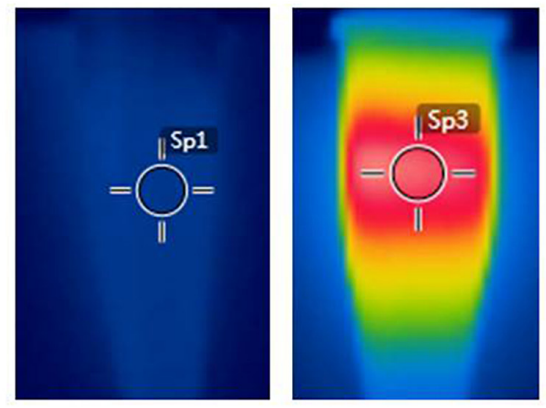

FIGURE 4 | The photothermal performance of the CuS@BSA nanoparticle. (A) Temperature changes of water and CuS@BSA nanoparticle aqueous solution at different concentrations with irradiation time. (B) The temperature changes of the CuS@BSA nanoparticle aqueous solution (90 $\mu \mathrm{g} / \mathrm{mL})$ for $180 \mathrm{~s}$ with NIR laser $\left(1064 \mathrm{~nm}, 0.73 \mathrm{~W} / \mathrm{cm}^{2}\right)$ and then the laser was shut off. (C) Plot of temperature change $(\Delta \mathrm{T})$ over a period of $180 \mathrm{~s}$ vs. the concentration of CuS@BSA nanoparticle. (D) The infrared images of CuS@BSA nanoparticle aqueous solution. Left and middle images were the infrared images of CuS@BSA nanoparticle aqueous solution in tube before and after being irradiated by NIR laser, respectively. The right image was the scale bar of temperature. The color represented various temperature. Sp1 and Sp3 were marked as the points that the temperature were recorded.

cell viability. For the case of HeLa cells treated for $24 \mathrm{~h}$, the cell viability remained above 90\% when the applied CuS@BSA nanoparticle concentrations ranged from 0.7 to $22.5 \mu \mathrm{g} / \mathrm{mL}$ for 24 and $48 \mathrm{~h}$. It was worth noting that there was a special phenomenon once the cells were treated with the CuS@BSA nanoparticle at the concentration of $45 \mu \mathrm{g} / \mathrm{mL}$. When the treatment time was $24 \mathrm{~h}$, the cell viability remained above $80 \%$ (Figure 2A). When the treatment time was prolonged to $48 \mathrm{~h}$, the cell viability was only about 35\% (Figure 2B), which indicated that the CuS@BSA nanoparticle was a concentrationand time-dependent cytotoxicity pattern in the cell viability.

\section{Cell Apoptosis Assay After Co-cultured With CuS@BSA Nanoparticle}

To systematically assess the toxicity of the CuS@BSA nanoparticle on cells, the fraction of apoptotic cells caused by the nanoparticle were analyzed by flow cytometry (Figure 3 ). Here, only the cells treated with nanoparticles at a higher concentration were investigated. For the case of HeLa cells treated with the CuS@BSA nanoparticle at a concentration of 22.5 and $45 \mu \mathrm{g} / \mathrm{mL}$ for $24 \mathrm{~h}$, the cell apoptosis rates of the CuS@BSA nanoparticle in all groups were comparable to that in the control groups. When the co-incubation time was extended to
$48 \mathrm{~h}$, the cell apoptosis rates showed no obvious change, while the HeLa cells were treated with the CuS@BSA nanoparticle at the concentration of $22.5 \mu \mathrm{g} / \mathrm{mL}$. However, when the concentration of the CuS@BSA nanoparticle was increased to $45 \mu \mathrm{g} / \mathrm{mL}$, the cell apoptosis rates increased dramatically to $29.22 \%$.

\section{The Photothermal Performance}

The strong NIR absorption of the CuS@BSA nanoparticle motivated us to evaluate their photothermal conversion ability. The quantitative temperature changes of the CuS@BSA in an aqueous solution at different concentrations with time were measured.CuS@BSA nanoparticle was dispersed in water at concentrations ranging from 22.5 to $180 \mu \mathrm{g} / \mathrm{mL}$, and then irradiated with a $1064 \mathrm{~nm}$ laser (a continuous wave fiber-coupled laser, $0.73 \mathrm{~W} / \mathrm{cm}^{2}$ ) for $180 \mathrm{~s}$. Pure water was used as a control. As shown in Figure 4A, the temperatures of all the CuS@BSA nanoparticle samples increased with the irradiation time, and the temperature increased more rapidly when increasing the concentration of CuS@BSA nanoparticle. The photothermal conversion efficiency of CuS@BSA nanoparticles used in this work was $69.37 \%$. After irradiation for $180 \mathrm{~s}$, the temperature of the CuS@BSA nanoparticle aqueous solution was increased by $40^{\circ} \mathrm{C}$ at a concentration of $180 \mu \mathrm{g} / \mathrm{mL}$ (Figure 4C). 


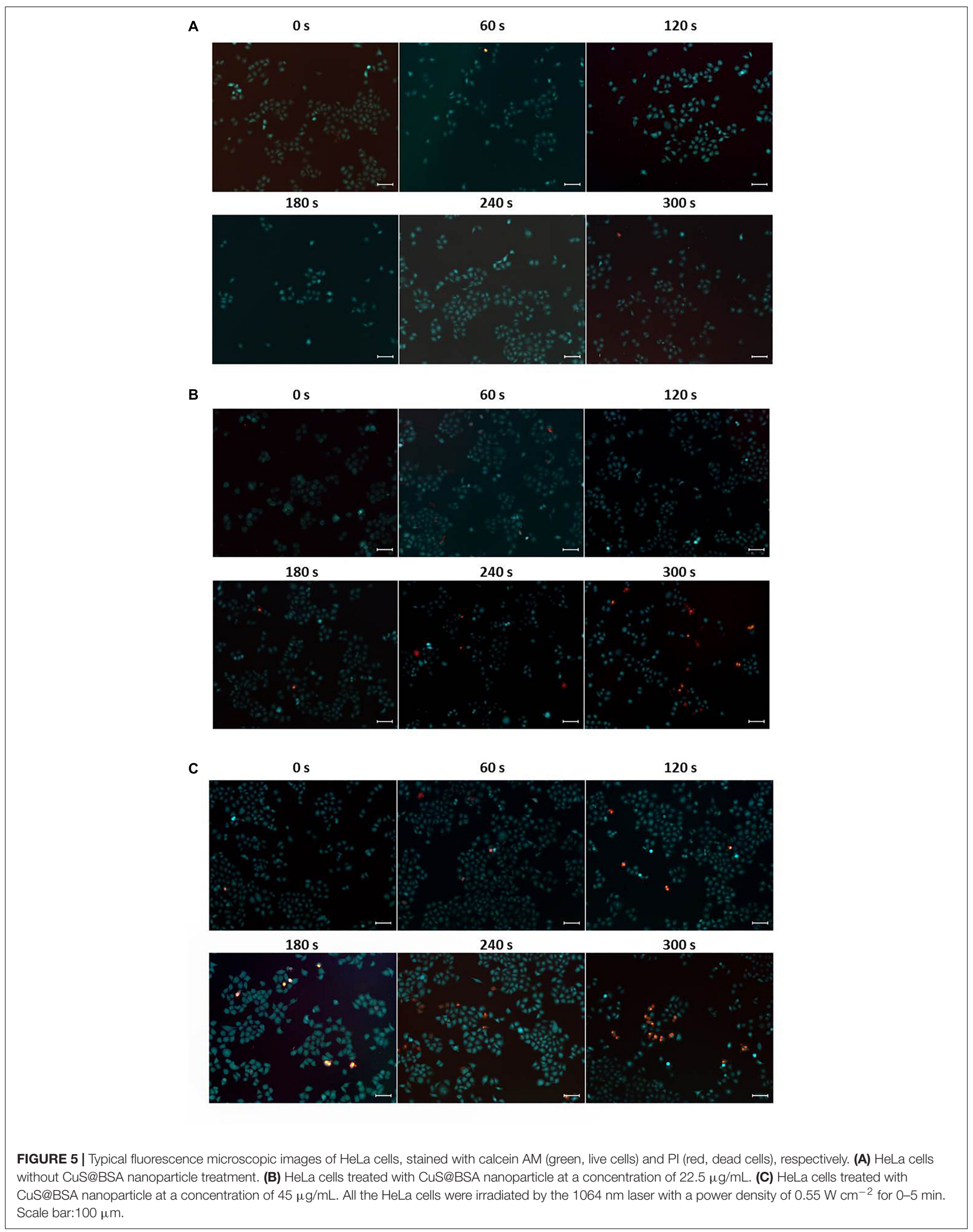




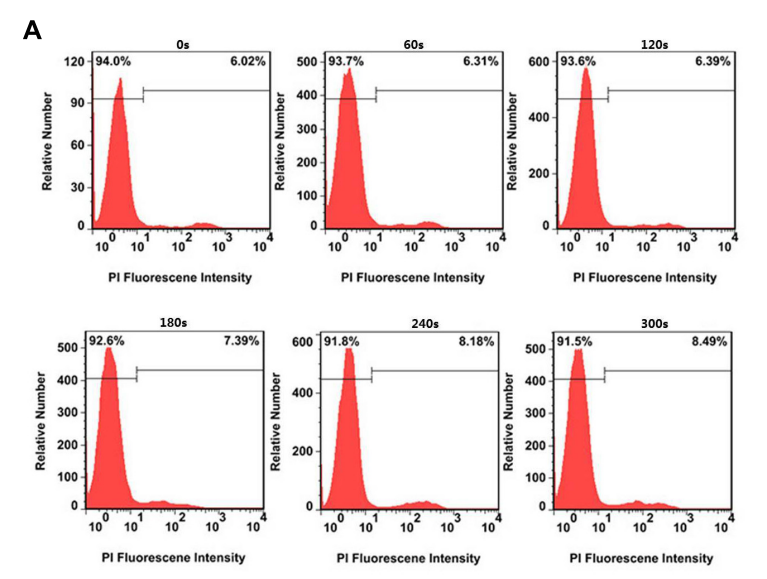

B
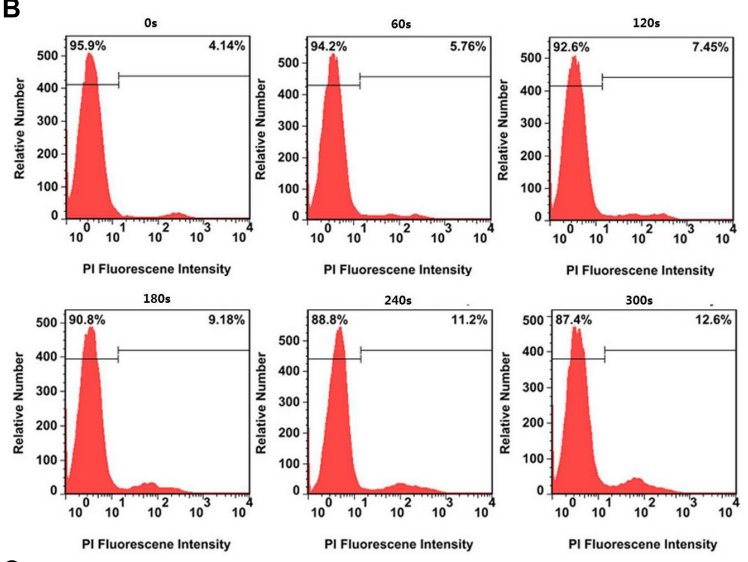

C
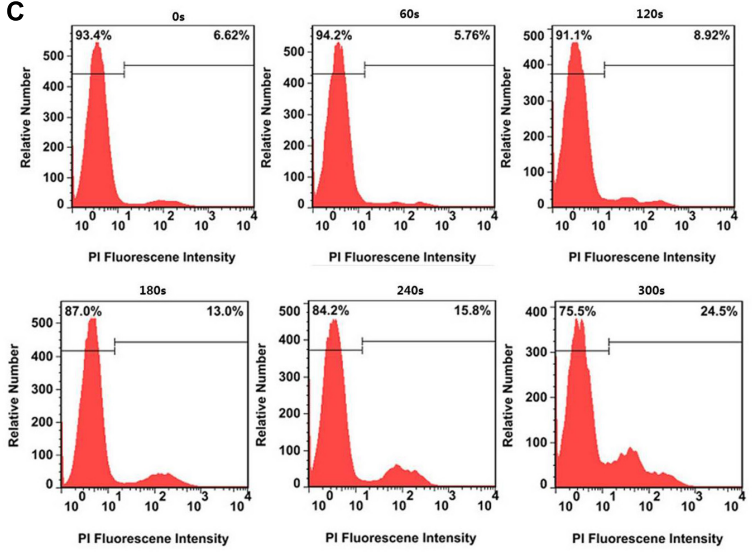

FIGURE 6 | Typical cell viability after treatment without and with CuS@BSA nanoparticles over irradiation time. (A) HeLa cells treated with different laser irradiation time without CuS@BSA nanoparticles. (B) HeLa cells treated with the CuS@BSA nanoparticle at a concentration of $22.5 \mu \mathrm{g} / \mathrm{mL}$. (C) HeLa cells treated with the CuS@BSA nanoparticle at a concentration of $45 \mu \mathrm{g} / \mathrm{mL}$. All the HeLa cells were irradiated by the $1064 \mathrm{~nm}$ laser with a power density of $0.55 \mathrm{~W} \mathrm{~cm}^{-2}$ for $0-5 \mathrm{~min}$.

\section{In vitro Photothermal Efficiency of CuS@BSA Nanoparticle}

The high photothermal conversion efficiency and low cytotoxicity of CuS@BSA nanoparticle prompted us to evaluate

their feasibility as a PTT agent for cancer therapy. The HeLa cells co-cultured with the CuS@BSA nanoparticle were irradiated by $1064 \mathrm{~nm}$ laser. The inverted luminescence microscope was used to distinguish the live cells and dead cells qualitatively. The flow cytometry analysis was applied to calculate quantitatively the cell viability. According to the in vitro toxicity results, we chose two concentrations, 22.5 and $45 \mu \mathrm{g} / \mathrm{mL}$, for PTT cancer cell treatment. The microscopic images showed that the irradiating laser had no obvious destructive effects on the HeLa cells without CuS@BSA nanoparticle treatment, even after being irradiated for $360 \mathrm{~s}$ (Figure 5A). However, the live cells decreased and the dead cells increased over the irradiation time while the HeLa cells were treated with CuS@BSA nanoparticles (Figures 5B,C). In comparing the fluorescence images of cells treated with CuS@BSA at different concentration levels but for the same amount of irradiation time, no obvious differences were observed.

The flow cytometry analysis provides the quantitative photothermal efficiency of CuS@BSA nanoparticle for in vitro cell PTT. The results showed that the dead cells increased slightly, from 6.02 to $8.49 \%$, while the HeLa cells were treated without nanoparticles over the irradiation time (Figure 6A). When the HeLa cells were treated with the CuS@BSA nanoparticle at a concentration of $22.5 \mu \mathrm{g} / \mathrm{mL}$, the dead cell percentage increased significantly, from 4.14 to $12.6 \%$ (Figure 6B). When the HeLa cells were treated with the CuS@BSA nanoparticle at a concentration of $45 \mu \mathrm{g} / \mathrm{mL}$, the dead cell percentage increased dramatically, from 6.62 to $24.5 \%$ (Figure $6 \mathrm{C}$ ).

\section{DISCUSSION}

In recent years, many methods for synthesizing the $\mathrm{CuS}$ nanoparticle have been developed, and the toxicity of $\mathrm{CuS}$ nanoparticle has also been studied. However, the synthetic methods currently known are complicated, and the toxicity study on $\mathrm{CuS}$ nanomaterial is not clear enough. Therefore, this study used a protein BSA as a coating material to synthesize the $\mathrm{CuS}$ nanoparticle in a fast and simple way, and systematically studied the toxicity and its photothermal performance of this nanoparticle.

It is well known that BSA is an excellent biomolecule for nanoparticle surface modification due to its large quantity, low cost, and good biocompatibility. The BSA contains 35 potential thiol groups, including 17 disulfide bonds and 1 free cysteine, which can be used to stabilize the affinity of CuS nanoparticle due to the strong binding between $\mathrm{Cu}$ and thiol groups (Zhang et al., 2015). Here we synthesized ultrasmall CuS@BSA nanoparticle in a fast and simple way. The TEM size was only around $8 \mathrm{~nm}$, which was suitable for further functionalization and applied in in vitro/in vivo biosensing, bioimaging and therapy. The NIR absorption of the CuS@BSA nanoparticle was at 1060, which was in the range of the biological tissue optical window $\mathrm{nm}$ and suitable for in vivo PTT applications.

The safety assessment of the nanoparticle is the most important thing before we started the biomedical application. In this work, the MTT and flow cytometry assays were performed 
to investigate the cytotoxicity and apoptosis of CuS@BSA nanoparticles. The results indicated that the toxicity of such nanoparticle was time- and concentration-dependent. The longer the co-incubation time and the higher the concentrations that were applied, the more cells were apoptosis-induced. There are two speculations to explain the cell apoptosis. One consideration was that that too much nanoparticle entered the cells and resulted in cells dysfunction (Guo et al., 2018), which further caused apoptosis. The other one was that the $\mathrm{Cu}^{2+}$ leakage induced apoptosis (Nordin et al., 2018). Further work should be carried out to investigate the appropriate pathway. The toxicity assessment could only provide a safe concentration range for biomedical application.

Generally, the photothermal conversion was nanoparticle concentration-dependent (Habash et al., 2007; Feng et al., 2015; Zhang et al., 2015). According to the cytotoxicity assay, CuS@BSA at the concentration of $22.5 \mu \mathrm{g} / \mathrm{mL}$ was the highest safe concentration for an in vitro cells study. In this work, the photothermal performance of the CuS@BSA nanoparticle in an aqueous solution at a concentration from 22.5 to $180 \mu \mathrm{g} / \mathrm{mL}$ was performed, in order to further choose the appropriate concentration range of nanoparticles under the safe premise. To our knowledge, it was the highest photothermal conversion efficiency of the CuS nanoparticle as a PTT sensitive agent at a similar CuS nanoparticle concentration (Feng et al., 2015; Zhang et al., 2015; Yang et al., 2016; Liang et al., 2018). In contrast with other reports, our results showed that the temperature would increase rapidly after treatment with minutesirradiation. Even at the lowest concentration, $22.5 \mu \mathrm{g} / \mathrm{mL}$, the temperature increase by $26^{\circ} \mathrm{C}$ after $180 \mathrm{~s}$ irradiation time. It has been reported that cancer cells can be killed after maintenance at $42^{\circ} \mathrm{C}$ for $15-60 \mathrm{~min}$ (Habash et al., 2007). The duration can be shortened to 4-6 min for temperatures over $50^{\circ} \mathrm{C}$. Thus, our CuS@BSA nanoparticle would reduce the irradiation time and has great potential as an excellent PTT agent candidate.

The in vitro PTT experiments verified the excellent photothermal conversion performance of the CuS@BSA. The PTT efficiency of the nanoparticles for HeLa cells was obvious irradiation time-dependent. In addition, over the same irradiation time, with the concentration increasing, the dead cell percentage also increased. The dead cell increased from 12.6 to $24.5 \%$ when the concentration of CuS@BSA increase from 22.5 to $45 \mu \mathrm{g} / \mathrm{mL}$. However, in order to obtain the better therapy efficiency, we should keep the balance between the cytotoxicity

\section{REFERENCES}

Balandeh, M., Mezzetti, A., Tacca, A., Leonardi, S., Marra, G., Divitini, G., et al. (2015). Quasi-1D hyperbranched WO3 nanostructures for low-voltage photoelectrochemical water splitting. J. Mater. Chem. A 3, 6110-6117. doi: 10.1039/C4TA06786J

Chen, L., Zhong, X., Yi, X., Huang, M., Ning, P., Liu, T., et al. (2015). Radionuclide 131 I labeled reduced graphene oxide for nuclear imaging guided combined radio- and photothermal therapy of cancer. Biomaterials 66, 21-28. doi: 10.1016/j.biomaterials.2015. 06.043 and the photothermal conversion and choose the appropriate concentration of the nanoparticles.

\section{CONCLUSION}

In summary, we have reported a novel strategy to synthesize the biocompatible CuS@BSA nanoparticle for in vitro PTT application. The characterization results showed that the size of the CuS@BSA nanoparticle particle was $8 \mathrm{~nm}$, which was suitable for both in vitro and in vivo biomedical applications. The CuS@BSA was not toxic for HeLa cells at a concentration lower than $22.5 \mu \mathrm{g} / \mathrm{mL}$ for $48 \mathrm{~h}$, but the critical apoptotic events occurred in HeLa cells after co-incubation with $45 \mu \mathrm{g} / \mathrm{mL}$ CuS@BSA for 48 h. The photothermal experiments demonstrate that our CuS@BSA nanoparticle is characterized with a high photothermal conversion, a short irradiation time and a long recovery time. The photothermal effect of the CuS@BSA in an aqueous medium was concentration-dependent and timedependent. Thus, this work provided a fast and simple way to synthesize an ultrasmall biocompatible nanoparticle, CuS@BSA, which had an excellent photothermal conversion feature, and could be an excellent PTT agent candidate in future clinics.

\section{AUTHOR CONTRIBUTIONS}

XW designed the experiments, conducted the basic work, and prepared the initial draft. ML designed the method to synthesize the CuS@BSA nanoparticles. MM performed the microscopic imaging. DC assisted in preparing the cells. NW conducted the photothermal experiments. LL analyzed the MTT data and proposed the data interpretation. ZL provided assistance for laser using and parameters choosing. GL helped to analyzed the apoptosis data. XW provided the statistical analysis and edited the manuscript. GX supervised the work and revised the manuscript.

\section{FUNDING}

This work was supported by the National Natural Science Foundation of China (31671491, 81772002, and 21677102) and the Basic Research Foundation of Shenzhen (JCYJ20170817102634964).
Choi, W. I., Kim, J. Y., Kang, C., Byeon, C. C., Kim, Y. H., and Tae, G. (2011). Tumor regression in vivo by photothermal therapy based on goldnanorod-loaded, functional nanocarriers. ACS Nano 5, 1995-2003. doi: 10. 1021/nn103047r

Dreaden, E. C., Alkilany, A. M., Huang, X., Murphy, C. J., and El-Sayed, M. A. (2012). ChemInform abstract: the golden age: gold nanoparticles for biomedicine. Cheminform 41, 2740-2779. doi: 10.1039/c1cs15 $237 \mathrm{~h}$

Dutta, A., and Dolui, S. K. (2008). Preparation of colloidal dispersion of CuS nanoparticles stabilized by SDS. Mater. Chem. Phys. 112, 448-452. doi: 10.1016/ j.matchemphys.2008.05.072 
Feng, W., Nie, W., Cheng, Y., Zhou, X., Chen, L., Qiu, K., et al. (2015). In vitro and in vivo toxicity studies of copper sulfide nanoplates for potential photothermal applications. Nanomedicine 11, 901-912. doi: 10.1016/j.nano.2014.12.015

Geng, J., Li, K., Pu, K. Y., Ding, D., and Liu, B. (2012). Conjugated polymer and gold nanoparticle co-loaded PLGA nanocomposites with eccentric internal nanostructure for dual-modal targeted cellular imaging. Small 8, 2421-2429. doi: 10.1002/smll.201102353

Goel, S., Chen, F., and Cai, W. (2014). Synthesis and biomedical applications of copper sulfide nanoparticles: from sensors to theranostics. Small 10, 631-645. doi: 10.1002/smll.201301174

Guo, C., Ma, R., Liu, X., Xia, Y., Niu, P., Ma, J., et al. (2018). Silica nanoparticles induced endothelial apoptosis via endoplasmic reticulum stress-mitochondrial apoptotic signaling pathway. Chemosphere 210, 183-192. doi: 10.1016/j. chemosphere.2018.06.170

Habash, R. W., Bansal, R., Krewski, D., and Alhafid, H. T. (2007). Thermal therapy, Part III: ablation techniques. Crit. Rev. Biomed. Eng. 35, 37-121. doi: 10.1615/ CritRevBiomedEng.v35.i1-2.20

Huang, X., Elsayed, I. H., and Elsayed, M. A. (2010). Applications of gold nanorods for cancer imaging and photothermal therapy. Methods Mol. Biol. 624, 343-357. doi: 10.1007/978-1-60761-609-2_23

Jiang, Q., Xu, L., Chen, N., Zhang, H., Dai, L., and Wang, S. (2016). Facile synthesis of black phosphorus: an efficient electrocatalyst for the oxygen evolving reaction. Angew. Chem. Int. Ed. Engl. 55, 13849-13853. doi: 10.1002/ anie. 201607393

Kievit, F. M., and Zhang, M. (2011). Surface engineering of iron oxide nanoparticles for targeted cancer therapy. Acc. Chem. Res. 44, 853-862. doi: 10.1021/ar2000277

Liang, L., Peng, S., Yuan, Z., Wei, C., He, Y., Zheng, J., et al. (2018). Biocompatible tumor-targeting nanocomposites based on $\mathrm{CuS}$ for tumor imaging and photothermal therapy. RSC Adv. 8, 6013-6026. doi: 10.1039/C7RA12 $796 \mathrm{~K}$

Liu, H., Chen, D., Li, L., Liu, T., Tan, L., Wu, X., et al. (2011). Multifunctional gold nanoshells on silica nanorattles: a platform for the combination of photothermal therapy and chemotherapy with low systemic toxicity. Angew. Chem. Int. Ed. Engl. 50, 891-895. doi: 10.1002/anie.201002820

Liu, X., and Swihart, M. T. (2014). Heavily-doped colloidal semiconductor and metal oxide nanocrystals: an emerging new class of plasmonic nanomaterials. Chem. Soc. Rev. 43, 3908-3920. doi: 10.1039/c3cs60417a

Liu, Y., Ai, K., Liu, J., Deng, M., He, Y., and Lu, L. (2013). Dopamine-melanin colloidal nanospheres: an efficient near-infrared photothermal therapeutic agent for in vivo cancer therapy. Adv. Mater. 25, 1353-1359. doi: 10.1002/adma. 201204683

Nel, A., Xia, T., Mädler, L., and Li, N. (2006). Toxic potential of materials at the nanolevel. Science 311, 622-627. doi: 10.1126/science.1114397
Nordin, N., Samad, W. Z., Kardia, E., Yahaya, B. H., Yusop, M. R., and Othman, M. R. (2018). Controlled release electrochemical synthesis and cytotoxicity study of copper(II) nanoparticles in copper(II) decanoate complex. Nano Brief Rep. Rev. 13:1850048. doi: 10.1142/S1793292018500480

Ozbay, E. (2006). Plasmonics: merging photonics and electronics at nanoscale dimensions. Science 311, 189-193. doi: 10.1126/science.1114849

Song, G., Liang, C., Yi, X., Zhao, Q., Cheng, L., Yang, K., et al. (2016). Perfluorocarbon-loaded hollow Bi2Se3 nanoparticles for timely supply of oxygen under near-infrared light to enhance the radiotherapy of cancer. $A d v$. Mater. 28, 2654-2654. doi: 10.1002/adma.201504617

Yang, W., Guo, W., Le, W., Lv, G., Zhang, F., Shi, L., et al. (2016). Albuminbioinspired Gd:CuS nanotheranostic agent for in vivo photoacoustic/magnetic resonance imaging-guided tumor-targeted photothermal therapy. ACS Nano 10, 10245-10257. doi: 10.1021/acsnano.6b05760

Yavuz, M. S., Cheng, Y., Chen, J., Cobley, C. M., Zhang, Q., Rycenga, M., et al. (2009). Gold nanocages covered by smart polymers for controlled release with near-infrared light. Nat. Mater. 8, 935-939. doi: 10.1038/nmat2564

Yuan, H., Fales, A. M., and Vodinh, T. (2012). TAT peptide-functionalized gold nanostars: enhanced intracellular delivery and efficient NIR photothermal therapy using ultralow irradiance. J. Am. Chem. Soc. 134, 11358-11361. doi: 10.1021/ja304180y

Zhang, C., Fu, Y. Y., Zhang, X., Yu, C., Zhao, Y., and Sun, S. K. (2015). BSAdirected synthesis of CuS nanoparticles as a biocompatible photothermal agent for tumor ablation in vivo. Dalton Trans. 44, 13112-13118. doi: 10.1039/ c5dt01467k

Zhou, M., Zhang, R., Huang, M., Lu, W., Song, S., Melancon, M. P., et al. (2010). A chelator-free multifunctional [64Cu]-CuS nanoparticle platform for simultaneous Micro-PET/CT imaging and photothermal ablation therapy. J. Am. Chem. Soc. 132, 15351-15358. doi: 10.1021/ja106855m

Zhu, Z. G., and Su, G. (2013). Spin manipulations through electrical and thermoelectrical transport in magnetic tunnel junctions. Sci. China Phys. Mech. Astron. 56, 166-183. doi: 10.1007/s11433-012-4960-x

Conflict of Interest Statement: The authors declare that the research was conducted in the absence of any commercial or financial relationships that could be construed as a potential conflict of interest.

Copyright (C) 2019 Wan, Liu, Ma, Chen, Wu, Li, Li, Lin, Wang and Xu. This is an open-access article distributed under the terms of the Creative Commons Attribution License (CC BY). The use, distribution or reproduction in other forums is permitted, provided the original author(s) and the copyright owner(s) are credited and that the original publication in this journal is cited, in accordance with accepted academic practice. No use, distribution or reproduction is permitted which does not comply with these terms. 